\title{
Study of the Structure and Properties of Nanoparticles Cynara scolymus L. Encapsulated With Sodium Carboxymethyl Cellulose Polysaccharide
}

\author{
Muborak A. Tulyasheva, Tashkent Pharmaceutical Institute, Uzbekistan \\ Sobitjan Y. Inagamov, Tashkent Pharmaceutical Institute, Uzbekistan \\ Gafur I. Mukhamedov, Chirchik State Pedagogical University, Uzbekistan
}

\begin{abstract}
In recent years, the interests of researchers have increased towards nanodrugs based on nanoparticles. The main direction of this work was to study the physicochemical, structural properties, and stability of encapsulated nanoparticles with the components from medicinal plants. Nanoparticles were obtained from the extract of the medicinal plant Cynara scolymus L. using a metal salt. Initial experimental data have shown that the originally obtained nanoparticles were not stable. Therefore, the nanoparticles were then stabilized with added sodium carboxymethylcellulose polysaccharide. It was found that new nanoencasulated nanoparticles with compounds from medicinal plants are very stable and can be a source of effective nanodrugs based on them.
\end{abstract}

\section{KEYWORDS}

Encapsulation, Extract, Nanoparticles, Polysaccharide, Prickly Artichoke, Properties, Sodium Carboxymethylcellulose, Structure

\section{INTRODUCTION}

In the development of modern nanotechnology, a significant role is played by research on nanoparticles obtained from extracts of a medicinal plant using solutions of metal salts. This is due to a wide range of possibilities for their practical application, in which the specific properties of both the nanoparticles themselves obtained from the extracts of medicinal plants and the materials modified by them are used (Evstigneeva \& Pchelkin, 2006; Sergeev, 2003). Nowadays, the nanoscience developing at the intersection of physics, chemistry, and biology, where the rapid advance of the nanoscience in the last decade is primarily due to the development of new methods for synthesis, study, and modification of nanostructures (Sergeev, 2003). In addition, nanoparticles can be classified by their size, number of atoms in a particle, and number ratio of surface to bulk atoms. In nanoscience the key problem is to determine the influence exerted by the size of particles on their chemical activity. To find it, the methods for synthesis and stabilization of nanoparticles are important (Sergeev, 2003). Methods for analysis of the size and properties of nanoparticles are also very important (Sergeev, 2003).

In addition to the above, in recent years, researchers have increasingly put the attention to the creation of nanodrugs based on nanoparticles (nanoparticles are the sizes of medicinal particles at nanoscale levels, i.e. a billionth of a meter), which will be delivered by blood flow directly to the 
diseased human organ, which will increase the efficiency of its use and reduce side effects. In this regard, obtaining effective nanodrugs based on natural raw materials is relevant (Egorova, 2011a; Egorova, 2011b).

Among the methods for producing nanoparticles, a large group is formed by methods of chemical synthesis based on the reduction of metal ions to atoms in solutions under conditions favorable to the subsequent aggregation of atoms and ions with the formation of nanoparticles. One of these methods of obtaining metal nanoparticles is the method of biochemical synthesis, on the basis of which a new direction has emerged in the field of synthesis, research of properties and development of applications for metal nanoparticles. It can be said that the need to create such a direction stemmed from the needs for the development of research in nanochemistry, nanomedicine and nanopathology, focused primarily on solving applied problems using the achievements of nanotechnology (Egorova, 2011a).

The possibilities of studying the properties of metal nanoparticles, developing options for their practical application, as well as elucidating the mechanisms of their biological action largely depend on the method of preparation, which in many cases determines their structure, size, physical and chemical properties and, most importantly, stability - the lifetime in nanoscale state (Egorova, 2011a).

Thus, it can be argued that the determination of the ways and means of the effect of metal nanoparticles on a living organism is an extremely important and urgent work, which is necessary, firstly, to improve existing and create new drugs or methods of treatment, that is, for nanomedicine, in secondly, to clarify the causes of nanopathologies and, thirdly, to establish scientifically grounded permissible ranges of concentrations and sizes of nanoparticles in water, air, or in the composition of various materials with which a person comes into contact (Egorova, 2011b; Scientific foundations and prospects for the development of oncology, 2008).

In this regard, the main goal of this work is to study the physicochemical, structural properties and stability of encapsulated nanoparticles obtained from the medicinal plant of the prickly artichoke - "Cynara scolymus" and the development of new effective nanodrugs based on them.

\section{EXPERIMENT}

\section{Materials And Methods}

In this work, nanoparticles obtained from the medicinal plant artichoke prickly - "Cynara scolymus" are selected as the object of research. To obtain nanoparticles from "Cynara scolymus" prepared an aqueous-alcoholic extract from the leaves of prickly artichoke in the ratio: dry artichoke: $70 \%$ ethyl alcohol $=1: 10$. Nanoparticles were obtained from the extract with the introduction of metal salts of various concentrations (from $0.1 \%$ to $5 \%$ ) by the method of biochemical synthesis. It should be noted that, in comparison with other chemical methods, biochemical synthesis has a number of advantages that are important for the practical application of metal nanoparticles. First, in biochemical synthesis, the formation of nanoparticles occurs in solution, while in many other methods to obtain nanoparticles, it is necessary to create a vacuum or an atmosphere of an inert gas. This circumstance, as well as other features of the synthesis procedure, allow avoiding the complications necessary in other methods, which significantly reduce the cost of the final product. Second, it turns out to be possible to obtain nanoparticles that are stable in solution for a long time, which is very important both for the study of their properties and structure, and for applied developments. Third, for some metals it is possible to increase the degree of conversion of metal ions into nanoparticles in comparison with that achieved by other methods. Fourth, the method of biochemical synthesis is more environmentally friendly. The nanoparticles obtained by the method of biochemical synthesis from the extract of prickly artichoke - "Cynara scolymus" had a spherical shape with an average particle size of 50 - $120 \mathrm{~nm}$ (Mirrakhimova \& Yunuskhodzhaev, 2013; Mirrakhimova \& Yunuskhodzhaev, 2014; Mirrakhimova \& Yunuskhodzhaev, 2015). 
Synthesis of nanoparticles from the extract of prickly artichoke Cynara scolymus is performed at room temperature, by stirring the extract until the color changes. In the course of the experiment the formation of turbidity of the extract solution and further precipitation of particles was observed for a certain time by spectrophotometry, which indicated the formation of nanoparticles of magnesium metals. Magnesium sulfate $\left(\mathrm{MgSO}_{4}\right)$ was used in this study as a metal salt which facilitates the formation of nanoparticles. Magnesium sulfate ("Magnesii sulfatis" - another name for Epsom salts, magnesia, etc.) is a chemical that applies in medicine to cure the magnesium deficiency, and which contains magnesium ions and ions of the sulfate group as active ingredients. $\mathrm{MgSO}_{4}$ forms colorless rhombic crystals and has molecular weight (in a.m.u.) - 120.36. (Egorova, 2011a; Egorova, 2011b).

In the obtained extracts from prickly artichoke, aggregation occurs over time. To prevent aggregation of the resulting nanoparticles, stabilizers are used in all cases, which ensure the stability of the system. Stabilizers are usually polymers of natural origin - polysaccharides, gelatin, starch, agar-agar, etc. or synthetic polymers and surfactants. In this work, to enhance the aggregate stability, we used the polysaccharide sodium carboxymethyl cellulose (Na-CMC). For this, solutions were prepared in various ratios of Na-CMC: extract "Cynara scolymus" = 80:20; 60:40; 40:60; 20:80.

The introduction of $\mathrm{Na}-\mathrm{CMC}$ largely prevents aggregation and reduces the average size of nanoparticles. Mixing a solution of prickly artichoke extract with Na-CMC forms nanoparticles (C $=0.1$ basic $*$ mol / l) in various volumetric ratios, in which the nanoparticles are stabilized by the polysaccharide $\mathrm{Na}-\mathrm{CMC}$.

\section{RESULTS AND DISCUSSIONS}

In pharmaceutical industry, the use of nanoparticles for the delivery of a drug to specific organ is considered promising and already applied in some medicines. In nanodrugs the drug molecules are grafted onto the nano-sized carrier creating nanoparticles with the drug incorporated. As a result of modification, nanoparticles make drug soluble in blood and nanosized particles are able to penetrate cell membranes. This facilitates the process of delivery of medicinal nanoparticles to the "target" - to the affected cells that require treatment. In addition, research on nanoparticles obtained from medicinal plant extracts using solutions of metal salts plays a significant role in the development of modern nanotechnology. This is due to a wide range of possibilities for their practical application, in which the specific properties of both the nanoparticles themselves obtained from the extracts of medicinal plants and the materials modified by them are used (Bharali et al., 2003; Neradovic et al., 2004).

It is known from the literature (Bharali et al., 2003; Neradovic et al., 2004) that the rate of formation, yield, size and stability of nanoparticles depend on various factors - the specific composition of metal salt, the concentration of metal salt, and the degree of hydration. Experimental data showed that with the increase in the initial concentration of the metal salt, an increase in the size of the resulting nanoparticles and the range of their size distribution is observed: the particle size from 120 to 250 changes for nanoparticles obtained with sodium, and for magnesium from 140 to $280 \mathrm{~nm}$ at a salt concentration of the solution from $0.1 \%$ to $5 \%$.

Thus, for the first time, nanoparticles were obtained from an aqueous-alcoholic extract of prickly artichoke Cynara scolymus using metal salts of various concentrations. It was found that with an increase in the concentration of salt solutions the rate of formation and the size of nanoparticles obtained from the extract of the medicinal plant artichoke prickly Cynara scolymus increases in direct rate/proportion.

To elucidate the interaction of Na-CMC nanoparticles - "Cynara scolymus" the IR spectroscopic method of analysis was applied. The IR spectra of the extract "Cynara scolymus" with nanoparticles and solutions of nanoparticles of metal salts in the presence of the polysaccharide Na-CMC of various ratios are given in Fig. 2. It should be noted that the IR spectra of all ratios of Na-CMC and the extract with different ratios had almost the same set of absorption bands and differed to a large extent in intensity from each other and in the shift of some absorption bands. The structure of the 


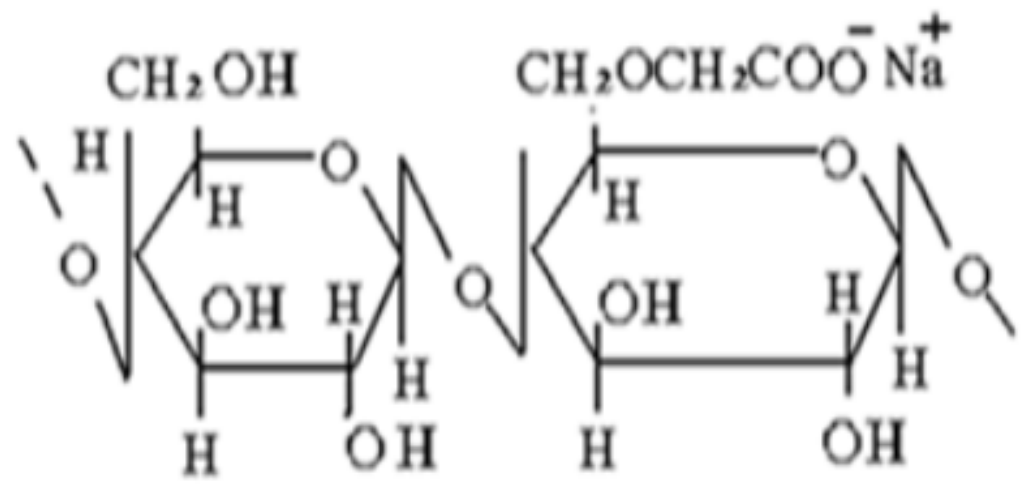

Figure 2. IR absorption spectrum of the starting product $\mathrm{Na}-\mathrm{CMC}$ at $\mathrm{pH}=6.8$

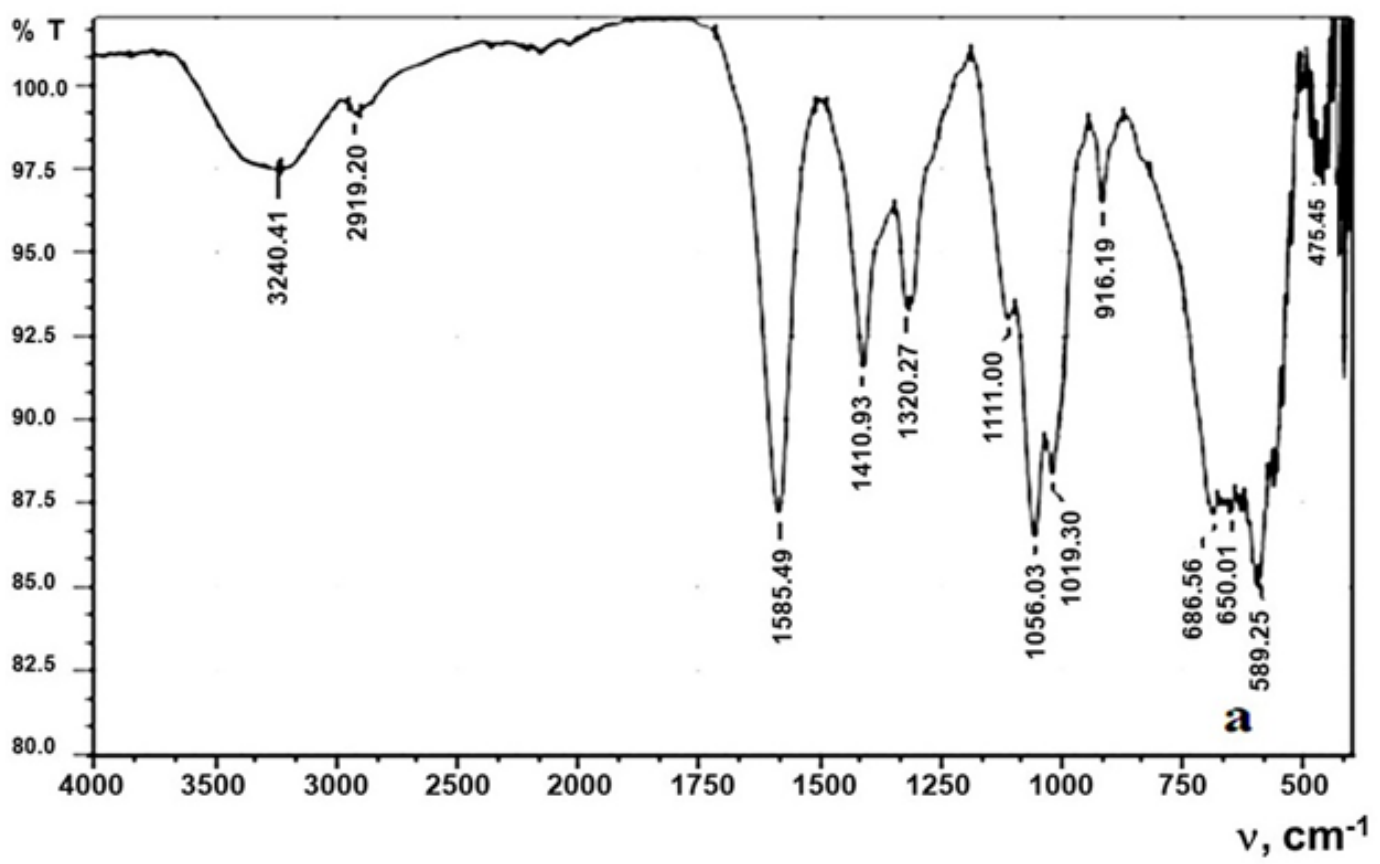

$\mathrm{Na}-\mathrm{CMC}$ products was established using IR spectroscopy methods and on data based on the literature data (Inagamov, 1914).

Now let us dwell on some of the structural features of Na-CMC that explain its properties. It is known that $\mathrm{Na}-\mathrm{CMC}$, in addition to the usual polydispersity for high-molecular compounds, has a significant compositional chemical heterogeneity (Inagamov \& Mukhamedov, 2011); has a different quantitative ratio of functional groups in the chain and a different distribution pattern of these groups in the chain. Therefore, it can be considered as a copolymer consisting of two types of units: D - glucopyranose with glucopyranose glycolic acid. In neutral media at a pH of about 7, both 
Figure 3. IR absorption spectrum of magnesium-based nanoparticles using an extract of the medicinal plant Cynara scolymus in relation to $\mathrm{Na}-\mathrm{CMC}$ : extract "Cynara scolymus" $=20: 80$

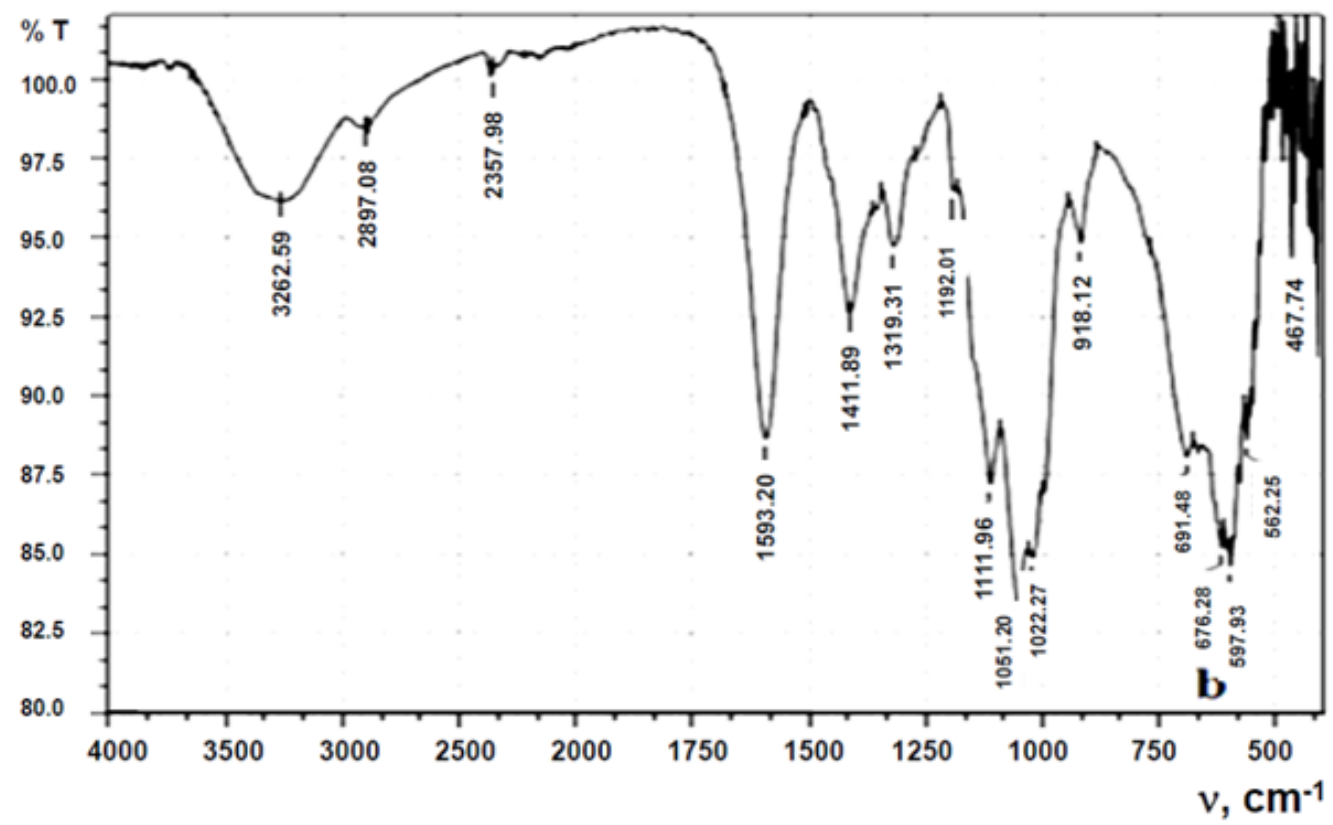

unsubstituted hydroxyl groups and a mixture of ionized carboxyl groups are present in the Na-CMC macromolecule (Fig. 1 and scheme):

A quantitative analysis of the spectra of $\mathrm{Na}-\mathrm{CMC}$ using data on the characteristic frequencies of individual functional groups (Inagamov et al., 2008; Inagamov et al., 2011; Khafizov, 2005; Khafizov et al., 2000) made it possible to assign all absorption bands and establish structural regularities.

Thus, the above results of the study of the initial Na-CMC show that it is polyfunctional; the presence of $\mathrm{COO}-, \mathrm{COOH}$ groups in their macromolecules gives these polymers the characteristic properties of polyelectrolytes.

In the obtained IR spectra of Na-CMC: "Cynara scolymus" different ratios (Fig. 2-5) showed the presence of $-\mathrm{OH}$ groups located in the region of $3240 \mathrm{~cm}^{-1}$, as well as $1585 \mathrm{~cm}^{-1}$ and $1410 \mathrm{~cm}^{-1}$ which belong to the carbonyl groups of Na-CMC. With an increase in the content of the extract "Cynara scolymus" with nanoparticles in a mixture shows the shift of these absorption bands to the high-frequency region, i.e. at the ratio: Na-CMC: extract "Cynara scolymus" $=20: 80-1593 \mathrm{~cm}^{-1}$; at a ratio of 40:60 - $1597 \mathrm{~cm}^{-1}$; and at a ratio of 60:40 $-1600 \mathrm{~cm}^{-1}$. Apparently, the shift of absorption bands located in the region of $1585 \mathrm{~cm}^{-1}$ indicates that the carbonyl group related to Na-CMC is strongly adsorbed on nanoparticles of magnesium metal, which leads to stabilization of the extract of prickly artichoke Cynara scolymus.

The above experimental data are confirmed by optical microscopic images of mixtures of NaCMC solutions: extract "Cynara scolymus" different ratios, which is shown in Fig. 6. The size of the nanoparticles was determined from the images, where nanoparticles sizes range from $120 \mathrm{~nm}$ to 280 $\mathrm{nm}$. It should be noted that the introduction of Na-CMC largely prevents aggregation and reduces the average size of nanoparticles (Fig. 6a). By displacing a solution of nanoparticles of an extract of prickly artichoke with solutions of $\mathrm{Na}-\mathrm{CMC}$ in different volume ratios, a nanocomposite was obtained, in which the nanoparticles were stabilized by the polysaccharide Na-CMC (Fig. 6 b). Let us briefly present the results of the study of the physicochemical properties of the synthesized nanocomposites 
Figure 4. IR absorption spectrum of magnesium-based nanoparticles using an extract of the medicinal plant Cynara scolymus in relation to $\mathrm{Na}-\mathrm{CMC}$ : extract "Cynara scolymus" = 40:60

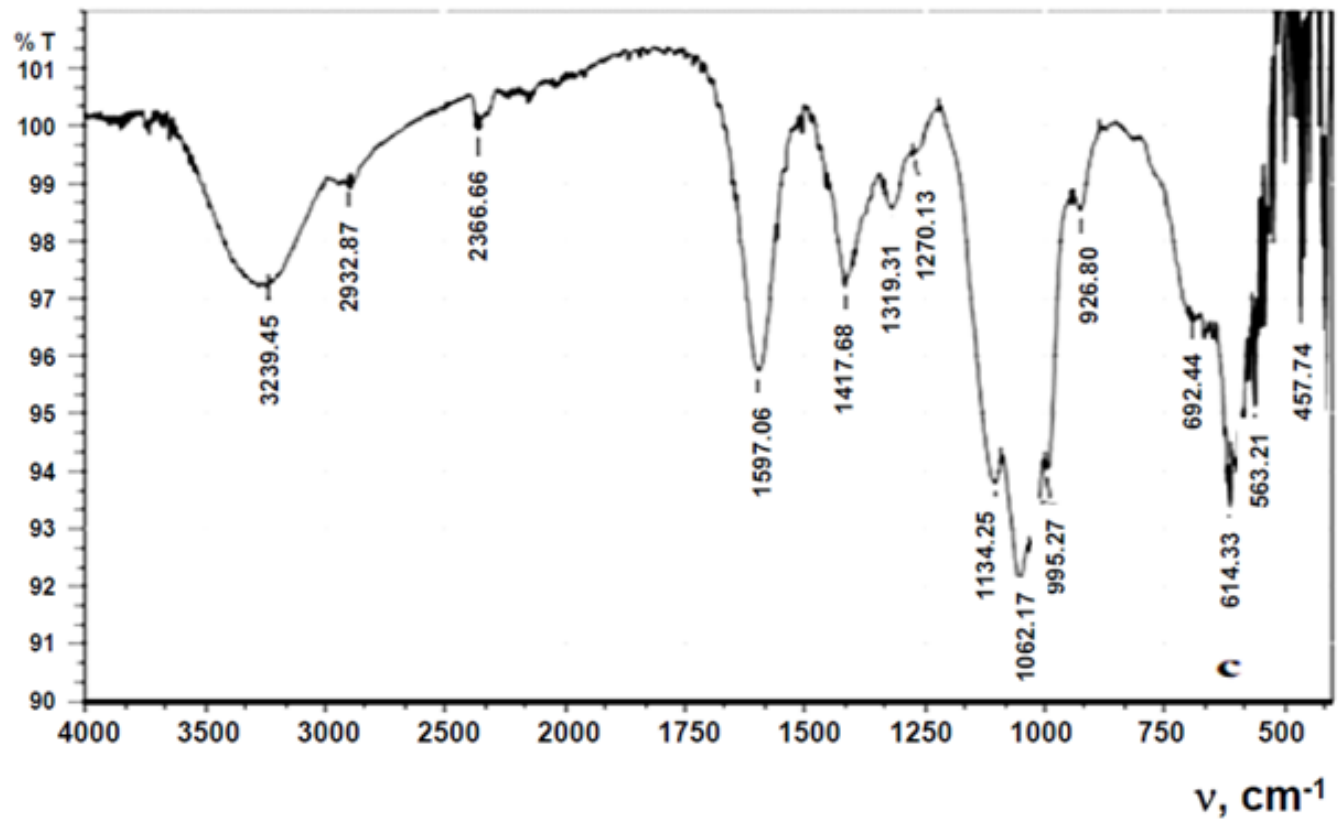

Figure 5. IR absorption spectrum of magnesium-based nanoparticles using an extract of the medicinal plant Cynara scolymus in the ratio Na-CMC: extract "Cynara scolymus" $=60: 40$

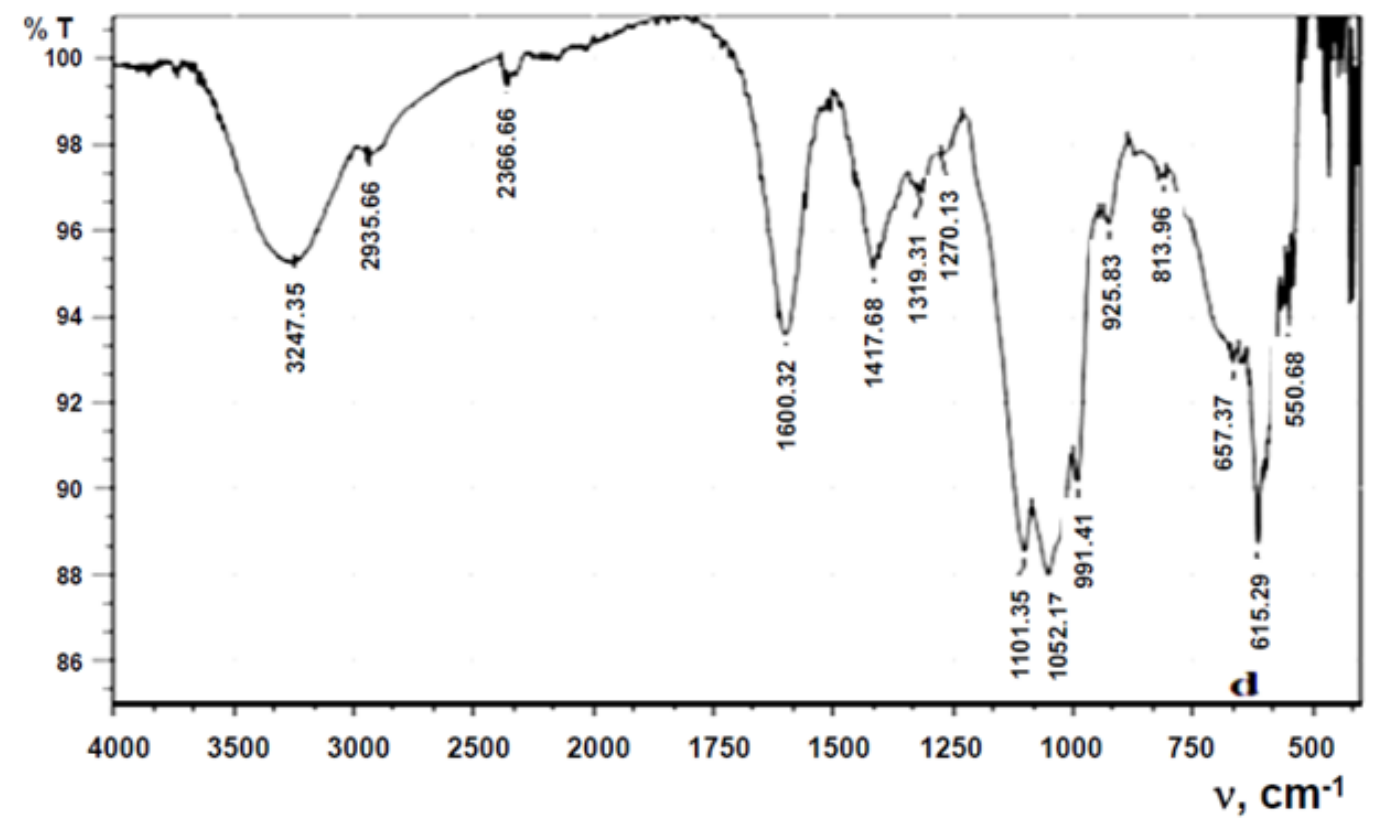


Figure 6. The mechanism of formation of nanoparticles from the extract of the medicinal plant Cynara scolymus (a) and microscopic image of encapsulated extract nanoparticles "Cynara scolymus" (b)

\section{With the introduction of}

\section{metal salts, nanoparticles} are formed

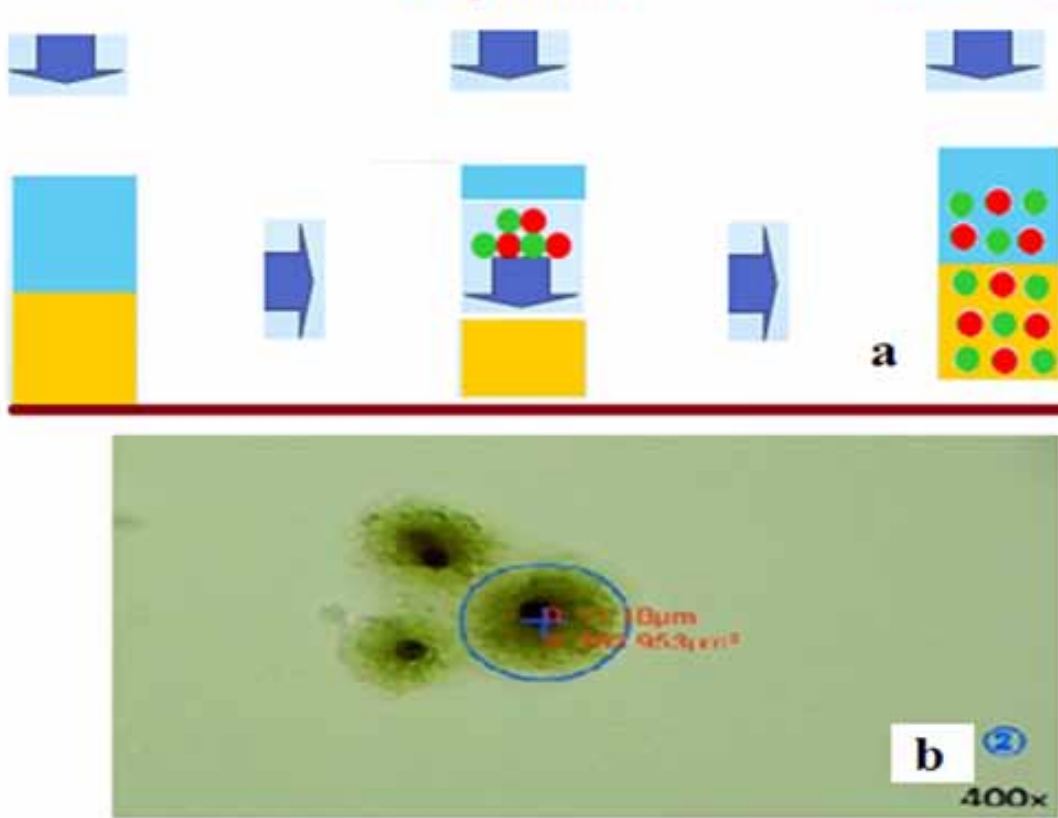

With the introduction of the polysaccharide, nanoparticles are stabilized Nanoparticle Deposition 


\section{CONCLUSION}

The main goal of this work was to study the physicochemical, structural properties and stability of encapsulated nanoparticles obtained from the medicinal plant of the prickly artichoke Cynara scolymus and the development of new effective nanodrugs based on them. In this regard, nanoparticles obtained from this medicinal plant were selected as the object of research.

To conclude, the synthesis of nanoparticles from the extract of the medicinal plant Cynara scolymus with the introduction of a magnesium sulfate salt is performed successfully. By varying the concentration of the polysaccharide Na-CMC, the aggregately stable nanocomposite was obtained, in which the nanoparticles were stabilized by the polysaccharide Na-CMC. 


\section{REFERENCES}

Anwar, W., \& Khitab, A. (2019). Nanotechnology From Engineers to Toxicologists: Risks and Remedial Measures. International Journal of Applied Nanotechnology Research, 4(2), 1-25. doi:10.4018/IJANR.2019070101

Bharali, D. J., Sahoo, S. K., Mozumdar, S., \& Maitra, A. (2003). Cross-linked polyvinylpyrrolidone nanoparticles: A potential carrier for hydrophilic drugs. Journal of Colloid and Interface Science, 258(2), 415-423. doi:10.1016/ S0021-9797(02)00099-1 PMID:12618113

Egorova, E. M. (2011a). Metal nanoparticles in solutions: biochemical synthesis, properties and applications. Abstract of a dissertation on the competition. scientific degree of DSc.

Egorova, E. M. (2011b). Interaction of silver nanoparticles with biological objects: Antimicrobial properties and toxicity for the other living organisms. Journal of Physics: Conference Series, 247, 012050. doi:10.1088/1742$6596 / 291 / 1 / 012050$

Evstigneeva, R.P., \& Pchelkin, V.P. (2006). Ligands of biologically active substances in silver and gold nanochemistry. Chemical and Pharmaceutical Journal, 40, 34.

Inagamov, S. Y. (1914). Development of technology for polycomplex pharmaceutical composites based on sodium carboxymethyl cellulose (Dissertation). IONKH AN RUz.

Inagamov, S.Y., \& Mukhamedov, G.I. (2011). Structure and physical-mechanical properties of interpolymeric complexes based on sodiumcarboxymethylcellulose. Journal of Applied Polymer Science, 122(3), 1749-1757.

Inagamov, S.Y., Mukhamedzhanova, M.Y., \& Mukhamedov, G.I. (2008). Rheological properties of polycomplex gels of sodium carboxymethylcellulose with urea-formaldehyde oligomers. Journal of Applied Chemistry, 81(2), 50 - 55.

Inagamov, S.Y., Mukhamedzhanova, M.Y., \& Mukhamedov, G.I. (2011). Polycomplex gels based on Na-CMC new prolongers of drugs. Chemistry of Plant Raw Materials, 1, 61-65.

Khafizov, M.M. (2005). Structures of interpolymer complexes based on carboxymethylselluljse and ureaformaldehyde resin. Crystallography Reports, 50(1), 94-97.

Khafizov, M. M., Mukhamedov, G. I., Karimov, Z. S., \& Akhmedzhanov, G. (2000). Interpolymer complexes in solving environmental problems and determining their structures by IR spectroscopy. Collection of scientific articles “INFRA-2000”, 181-183.

López, C. Y. T., Bueno, J. D. J. P., Torres, I. Z., López, M. L. M., Macias, A. H., \& Álvarez, J. E. U. (2019). $\mathrm{TiO}_{2}$ Nanotubes Transformation Into 4nm Anatase Nanoparticles: Anodizing Industrial Recycled Titanium for Photocatalytic Water Remediation. International Journal of Applied Nanotechnology Research, 4(2), $26-44$. doi:10.4018/IJANR.2019070102

Mirrakhimova, T.A., \& Yunuskhodzhaev, A.N. (2013). Study of lipid and amino acid composition of prickly artichoke leaves. Pharmaceutical Journal, 3, 23-27.

Mirrakhimova, T.A., \& Yunuskhodzhaev, A.N. (2014). The quantitative content of the main groups of biologically active substances in the leaves of prickly artichoke. Pharmaceutical Journal, 2, 41-45.

Mirrakhimova, T. A., \& Yunuskhodzhaev, A. N. (2015). The prickly artichoke is a promising medicinal plant. Chulpan Publishing House.

Neradovic, D., Soga, O., Van Nostrum, C. F., \& Hennink, W. E. (2004). The effect of the processing and formulation parameters on the size of nanoparticles based on block copolymers of poly (ethylene glycol) and poly (N-isopropylacrylamide) with and without hydrolytically sensitive groups. Biomaterials, 25(12), 2409-2418. doi:10.1016/j.biomaterials.2003.09.024 PMID:14741606

Scientific foundations and prospects for the development of oncology. (2008). In Nanotechnology and nanomaterials in medicine. Collection of materials of the XIX (82) session of the General Meeting of the Russian Academy of Medical Sciences. JSC Publishing House.

Sergeev, G. B. (2003). Nanochemistry. Moscow University Publisher. (in Russian) 
Smanova, Z. A., Rakhimov, T. K., Mukhamediev, M., Gafurova, D. A., \& Shaxidova, D. A. (2020). Possibilities of the Quantitative Comparison of Catalytic Capacity in Autocatalytic Processes of Palladium-Containing Nanocatalysts. International Journal of Applied Nanotechnology Research, 5(1), 35-46. doi:10.4018/ IJANR.20200101.oa4

Muborak A. Tulyasheva is a senior scientist and lecturer at the Department of Organic and Biological Chemistry, Tashkent Pharmaceutical Institute (Uzbekistan). She received her Ph.D. from the institute of the Chemistry of Plant Substances, Academy of Sciences of Uzbekistan.

Gafur Israilovich is a Doctor of Chemical Sciences, Professor, Rector of Chirchik State Pedagogical University (Uzbekistan). 